\title{
12. Mental Illness and Psychiatry Have Seen Substantial Change-But There is Still a Long Way To Go
}

\author{
Beverley Raphael
}

\section{The spectrum of health and illness}

The spectrum of what can be defined as health and illness has been progressively changed, by both the recognition of diverse diseases and how they are characterised, and the emergence of new patterns of problems. These new, emergent patterns of distress, impairment, 'strange' phenomena and suffering may be those associated with, for instance, a new infectious disease arising from viral mutation, or a new pattern of problems resulting from environmental or other hazards, or with socially driven behavioural change, or existing behaviour newly described, for instance, narcissistic personality disorder. Behavioural changes, whether the result of internally driven processes (such as chemical factors, e.g. neurotransmitters) or externally (as with adversity, violence, abandonment) may also lead to consistent patterns of suffering and dysfunction, which become defined as 'disorder'. Syndromes - patterns of symptomsmay constitute physical or psychiatric disorders that can be recognised and diagnosed as such and treated.

The consideration of the spectrum, or more specific patterns of symptoms that are categorised as illness, is important in identifying what is normal compared to what is pathological. The consideration of which symptoms, how many, for what duration, what levels of impairment are associated with disease or disorder, is important, but often difficult to separate from the human condition, particularly in terms of mental illnesses and disorders. This theme of the spectrum is important in considering change as it is relevant to psychiatry. It has been in recent years a rapidly advancing discipline involved with defining and classifying disorders, identifying causes, and providing effective treatment. But its interfaces with people's lives are often variable. The question of what is disorder is relevant to the changing conceptualisation of illness.

This is exemplified by debates about the suffering of grief, as for example over the loss of a loved one. Freud (1917) described the symptoms of mourning and melancholia and the normality of the former, along with the pathology of the latter. Lindemann (1944) described the symptomatology and management of 
acute grief. Engel (1961) asked 'Is grief a disease?' in a paper which argued that it was not. Many researchers in this field have continued to ask such questions, on issues including sadness and depression, shock, and post-traumatic stress disorders. And studies have evolved to lead to a recognition of prolonged grief, perhaps a disorder, a severe and disabling condition that could be treated-and currently argued in terms of its inclusion in the new edition of the Diagnostic and statistical manual of mental disorders of the American Psychiatric Association DSM V. At the same time others grieve for the loss of sadness as part of life and of human experience (Horwitz and Wakefield 2007).

This concept of life and human behaviour versus madness is highlighted when violence is addressed. When is this darker side of human nature part of the human condition, and when is it a psychiatric disorder? How can it be explained by syndromes of lack of control, rage disorders, or the consequence of psychosis, or response to perceived threat? When is the violent person not guilty because of mental illness - or more simply, when is that person bad, evil, a psychopath? The spectrum, the explanation, the definitions and naming, the environment, the purposes and effects of violence reflect the complexity of the life versus disorder questions.

The argument of the spectrum could continue - for instance, when does fear become an anxiety disorder? Or when do the multiple and prevalent psychoticlike experiences become an illness such as schizophrenia? When do passionate enthusiasms and driven activities become bipolar disorders, and when does forgetfulness in old age become dementia? When do the behavioural differences of children become disorders? These issues can be cogently argued against the diagnostic criteria developed from research and consensus. What is life and what is a disorder is much clearer when the differences are profound, but less so at the edges.

So the questions of change that arise here are those of the forces that have led to the recognition and effective treatment of many psychiatric or mental health problems, and how they deal with the boundaries of life versus disorder. At the same time, they call for research and knowledge development to find out why we have not or cannot prevent or cure the disorders that are so closely linked to our lives. 


\section{Societal and cultural dynamics of definition, diagnosis, prevalence and treatment}

Over time many societal and cultural dynamics have contributed to the naming, defining and treating of illnesses - from the 'humours' to plague and madness, to name a few. Understanding the causes and nature of problems of disease and sickness has developed through observation, efforts to deal with the condition, clinical or healing work, and science and knowledge that have progressively led to strategies for the definition, diagnosis and treatment of these forms of ill health.

Among diseases, psychiatric conditions - mental ill health - have been uncertain and complex, often poorly understood. Those dealing with the mentally ill were once known as alienists. Psychiatry has, however, changed to become a specialist medical discipline, one with its own professional training following a general medical degree, and its craft grouping, a college of like experts. In the years of its progressive change to this status, it has faced many challenges ranging from the stigma, fear, and discrimination experienced by mentally ill patients, and those who treat them, to the ebb and flow of anti-psychiatry movements.

Psychiatry is a complex discipline with multiple interfaces with other disciplines in related fields: psychology; general medicine where its separation from medical practice has led to the development of subspecialties such as consultationliaison psychiatry; paediatrics and child psychiatry; geriatric psychiatry; neuropsychiatry with neurology; and similarly neurochemistry and other biologics; and biological psychiatry. These subspecialisations continue to evolve as branches, but perhaps with less than optimal definition and multidimensional interactions for change.

It has often also been hypothesised that societal change influences the types of mental health problems that become prevalent, or are identified as prevalent. It was suggested that Freud's models, including the rise of psychoanalysis, were strongly influenced by the rise of physics. There is no doubt that earlier times, and even some currently, were principally informed by concepts of 'bad spirits'. War provides another example. 'Shell shock' affected soldiers of World War I, who were initially treated as cowards. This changed to 'combat fatigue' with World War II, and subsequently to post-traumatic stress disorder, which research has now defined, and for which it has delivered a number of scientifically valid treatments. Mild traumatic brain injury provides the most recent example of a related syndrome. Simple singular solutions are, however, infrequent. 
'Madness' or psychosis has been the most feared and stigmatised domain of psychiatric illness, with biological changes, the genetic variables revealed in the increased risk shown by family members, and the influence of environments such as cannabis use in the early teenage years. The rapid development of science for medication treatments has brought important changes in outcomes for many people suffering illnesses like schizophrenia. Nevertheless, fear and stigma continue. Perhaps the ultimate change is the role of people who are themselves ill in this way, having a 'Mad Pride' march to challenge other views.

The dominant models of psychiatric disorder are seen as based on Western cultural interpretations and values, and this is seen by some as a hegemony that is powerfully propagated.

Cultural understanding and belief have influenced the response to madness, in particular, psychosis. Formerly those affected were seen as having been taken over by an evil spirit and they were abandoned, chained up or locked away. In the earlier phases of recognition of this illness, this was also the pattern of care in what is now defined as the developed world. Change has occurred, even for those severely ill in this way, with the development of antipsychotic medications and other psychosocial interventions. These multiple treatment strategies have led to a life that can be lived in the community, not an institution of containment. However, for many with such severe illnesses, lifelong dependency, disability and dysfunction may persist so that the search for better treatment continues. One such valuable change has been the development of the 'recovery' movement, implemented in ways to empower those affected, and to bring a framework for belief, action, and hope.

The search for ways to prevent psychoses continues in research and service provision. There are now initiatives which seek to prevent the development of full-blown disorders by strategies which identify and treat those in the early stages of psychosis in the adolescent years. The Early Psychosis Prevention and Intervention Centre program 'EPPIC' (McGorry 2010) initiated in Australia is an important strategic change where the strong commitment of leaders in this field led to government commitment at policy, program and funding levels that supported widespread implementation.

The global need for change in the recognition of psychiatric disorders has been driven by the World Health Organization (WHO), with its focus on low and middle income countries. It has supported research to identify the extent and patterns of mental health need, i.e. mental illness, through initiatives such as the World Mental Health Survey; the reporting of policies, programs and service levels through the WHO Atlas project; as well as global programs supporting education, training and program development. Education and training to build expertise in low and middle income countries continues to 
be critical for change to more effective and humane treatments for those with severe psychiatric disorders. This extends from basic levels with primary care, through to psychiatric nurses and psychiatrists as well as psychologists and other allied health workers. The core essential provision of psychotropic medications is delineated and provided as far as possible. These changes, driven at a global level, continue to improve care and outcomes for those with severe mental illnesses. More specific focused programs to facilitate education and change include programs such as AsiaLink, an Australian/Asian program within which sits Asia Australia Mental Health, funded through both philanthropy and government. These programs have involved sophisticated educational initiatives with China and other Asian partnerships, achieving significant positive program development attuned to the defined needs of the participating countries.

One of the continuing challenges at a global level is the ongoing impact of adverse social determinants on mental health and wellbeing in all countries, but especially in low and middle income settings. These and other indices of adversity continue to have negative impacts, including those related to experience of violence, dislocation from home and country, and exposure to war and conflict. Increasingly in recent years, mass natural disasters such as earthquakes, hurricanes and cyclones, forest fires, drought, and floods have caused trauma, death and destruction, bringing adverse mental health consequences for significant numbers of affected populations. The response to these may also be influenced by the culture of belief in fate, acts of God, and acceptance of the specific cultural interpretations relevant to such events. Human-caused disasters such as terrorism have even more severe impacts for mental health.

Culture is, of course, central to how we interpret our world. The loss of cultural recognition and identity can add an extra burden of distress affecting mental health. The failure to recognise cultural values of the other may mean that their mental health needs are not understood. Nor may Western models of understanding or treatment be well received. Culturally informed practice is an important component of care. In Australia and other culturally diverse societies informed by multicultural programs, policies and service development can be attuned to cultural diversity. Recognition of our own culture and its beliefs and practices is also essential for developing true collaborations in service and care models. This change is progressive, from 'them' to 'us': the 'other', the 'alien', the 'unknown' can be perceived as threatening. 


\section{Mental health and mental illness across the lifespan}

As different cohorts - generations of baby boomers, X, Y, Z, and others - evolve over time, some generations are seen as having certain problems, and other factors also draw particular attention, such as the improvements in physical health and longevity.

Traditionally mental illness was seen in terms of psychosis, madness, and melancholia. Adult illnesses were, and still are, the principal focus. They are more overt, associated with heightened acute risk (e.g. violence, suicide) and onset in adolescent or early adult life. These are 'the mentally ill', suffering from, and diagnosed as having, 'serious mental illness'. This is, sadly still a defining term, rather than a person 'living with' a specific disorder.

With the ageing population there is concern for their mental health needs, especially proper recognition and treatment of their psychiatric disorders. Epidemiological research makes clear that there is a lower prevalence of psychiatric disorders generally in these years - but there is a high prevalence of dementia, with many in institutional care. The debate that remains is about in what category these illnesses of dementia sit, particularly whether or not they are psychiatric syndromes, as well as who is responsible for care. Research for prevention and treatment is extensive with these, as with any types of illness. As with other psychiatric disorders, comorbidity is frequent, for instance with depression, anxiety disorders, etc. Research-driven understandings are occurring, but these are chronic diseases and we have not yet found a way of change - by name or intervention - to make them go away, to prevent or cure them.

Conditions affecting children have had increasing attention, not necessarily because of a full understanding of the extent of their prevalence, but because of a growing body of research that makes it very clear that many, if not most, of the psychiatric problems of adult life begin from the earliest times. The role of the perinatal, early childhood and the early years is significant, with not only the genetic influences, but the gene-environment interactions, and the environments themselves.

The names given to disorders potentially excluded children from mental health or psychiatric assessment and care. Those with 'conduct disorder' were seen as being in need of other types of behavioural management, or discipline, as were those with other externalising conditions. The internalising, quiet behaviours were often seen as good, not causing problems. The progressive building of strong child psychiatry services, informed by a growing body of research in 
this field, has led to very significant developments. There have been excellent prevention and early intervention findings and randomised controlled trials of treatment for anxiety disorders, for instance.

Burgeoning research in the past decade has identified the vital role of early experiences of adversity in contributing in major ways to the development of mental disorders in childhood, adolescence and adult life, and even old age. These damaging experiences may occur in the spectrum 'family life difficulties'. Most likely to be associated with adverse health and mental health outcomes, however, are child abuse and neglect, especially child sexual abuse (penetrative) and other forms of violent abuse.

While studies also show the possibility of 'resilience' in the face of adverse environments, it is only in the most recent times that more population-based strategies have been implemented to build such strengths. 'Kids Matter', a universal primary school program, is one such evidence-based initiative that is currently being put in place across Australia. Triple P (Positive Parenting Pre-schoolers; Sanders 2013), developed in Australia, is established in many places internationally, but for multiple reasons not widely implemented in Australia. In addition, youth programs for adolescents such as 'Headspace' and the EPPIC early psychosis programs are being widely implemented on the basis of research and the costs of not changing, as established by research. Nevertheless, challenges to change come in many shapes and sizes, and from many sources: costs, lack of perceived benefits, and other political priorities, to name a few.

So across the lifespan of those who may develop a psychiatric disorder there are multiple challenges and changes - but the burden of mental ill health, measured at a population level, remains high.

\section{Models and systems of care}

Models and systems for the care and treatment of those with diagnosed mental disorders or mental illnesses have progressively changed and continue to do so. At earlier times, containment was the major management strategy for those who were ill, usually in asylums, which were large and often very dehumanised institutions. This was followed by deinstitutionalisation through the 1960s and 1970s, a movement that was facilitated by the availability of the new medications such as antipsychotics and antidepressants. Models of community care came into being. Nurses, psychiatrists and other professionals made home visits and community mental health centres were established. Psychiatric units were developed as part of general hospital services. 
These changes were seen as positive and valuable, although some elements of the old models of care persisted. These included the identification of people as 'the mentally ill', and as needing to be managed as though they could not know what was best for them. More importantly however, ideology continued the separation of mental health from health more broadly in terms of systems and the basis of care. 'Mental illness' programs, despite the growth of important services, often failed to address associated and frequent comorbid physical illness.

Systems of care changed in Australia, as in other countries, with the development and implementation of a national mental health policy and associated programs. These changes brought additional development with accountability, reporting requirements, funding base delineation, and health care utilisation statistics. Such policies are regularly updated and jurisdictions are engaged in implementing the proposed elements of service delivery. In addition to the progressive development of these frameworks, there have been improvements in services and outcomes for a great many of those affected. Regular data reporting of progress of change is provided through government agencies such as the Australian Institute of Health and Welfare.

Service systems and models of care, including specific hospital-based inpatient services, focus chiefly on those with 'serious mental illness', i.e. psychosis, severe depression and suicidal behaviour. Less severe cases were and are managed through community programs, for instance psychological counselling services, and primary care general practitioners. As indicated earlier there has also been growth in child and adolescent services.

Special program initiatives have also progressed, but not necessarily become formal parts of services systems and care delivery. Prevention programs, although well supported by research, remain a low priority despite their potential value. They do not have change drivers and traction, despite potential cost benefits, compared to youth programs focused on treatment such as 'Headspace' and the EPPIC early psychosis program which have had passionate drivers and changebased implementation.

Multidisciplinary teams have been a core component of mental health programs, bringing together diverse views in decision-making for clinical issues of concern. However, rather than reflecting the diverse expertise of different disciplines, at times there is still a culture of the core mental health professional or worker. Using a generic conceptualisation rather than specifying what is required has sometimes hindered opportunities for change and innovation. 
Service systems and models of care have continued to evolve and change in some ways, but not in others. The use of mental health units as specialist components of emergency departments and to provide emergency assessment, triage and management, sometimes occurred early (for example in the Royal Brisbane Hospital) but was resisted elsewhere because of the 'separation' ideology of mental health service models. Nevertheless such change has been driven forward and reflects a more modern, humane, less stigmatised process than the former model of the 'police van at the hospital back door'.

The separation of mental health from health, and of mental illness from the diverse and multiple specialised components of disease and illness management in the broader illness field, has reflected what could be considered a more limited and less sophisticated approach.

Specialised programs focused on different psychiatric disorders have only started to appear in recent years. And even though the costs and burden of these multiple psychiatric disorders is very significant, sophisticated, focused clinical programs such as those for cancer, heart disease and the like have not been well developed for mental health disorders. This is further compounded by the merging into chronic disease concepts - that illnesses like psychiatric disorders are not cured, require ongoing care, and are highly likely to recur. One may well ask where are the concepts of prevention and cure for psychiatry? Why are they so little believed in, and committed to, in our models and systems of care?

Hopeful, committed, skilled people advocate and work to improve mental health, and to lessen the human and social costs of mental illnesses. New systems and models continue to develop, such as mental health commissions, the 'recovery' movement, community care, and mental health online services. The evolution of change and its drivers occurs at multiple levels.

\section{Drivers of change}

Change continues to evolve for mental health, often from diverse processes. Further questions are: what change, why, and what are the outcomes? Does the change that occurs make things better or worse-better in terms of more positive health, lower costs, greater productivity, happiness or mental health? Or does it lead to greater suffering, increased pathology, impairment, and death? Our measures may not be adequate to answer these questions.

There are many drivers of change, and also resistances and barriers. Drivers include the ongoing pressures of a persistent problem, frustrations regarding the inability to access services at times of need and consistent 
pressures of severe demand. The issues themselves - such as the contributions of child abuse and neglect to mental ill health and suffering - may be drivers for both change and resistance, the latter coming from fear of the unfamiliar, the opening of Pandora's box. There are a number of influences driving change for mental health and for psychiatry.

\section{Science, its processes and outcomes}

It is clear that the findings of scientific research in the mental health field have been, and continue to be, very powerful influences. These have ranged from those leading to the development of effective medications to treat psychiatric disorders to the systematic exploration of symptom patterns, syndromes and their change over time, providing a shared base for understanding (as reflected in the World Health Organization's International Classification of Disease and the Diagnostic and statistical manual of the American Psychiatric Association).

The labelling and naming of disorders may change over time as they are further understood, as reflected in the trauma syndromes, depression, typologies of psychoses, autism spectrum, and attention deficit hyperactivity disorder (ADHD). The passionate curiosity of scientists in this field, the search to know to the fullest degree the brain and the mind and their relationships to pathology, has led to rapid advances in neuroscience as relevant to psychiatric disorders. Genomics, the chemistry of the brain and its transmissions, and more recently the recognition of neuroplasticity, have been important. There are also aims of enhancing understanding of and response to psychiatric disorders through prevention and treatment. The extensive programs from molecular psychiatry to translational psychiatry (Licinio 2013) aim to bring science to the coalface of treatment and care.

The findings of science are widely propagated through publication, conferences and the like, building the knowledge base. But essential to change is the integration of relevant research into the real world of treatment, to make mental health better. Translation, integration and embedding research into the understanding of psychiatric disorder is critical in driving change for the future. Major systems of research such as the US National Institute of Mental Health contribute to such research/change processes, the more so when linked to service delivery programs.

The rapidity of scientific development in this field, both basic science research, and social and other sciences, leads to a critically evolving challenge. How can we utilise the future developments of these fields, of society, of science, of technology, to address the rapidly advancing 'rolling front end' of problems and need, as suggested by the World Health Organization's Norman Sartorius in 1994. Future focus is critical for effective change. 


\section{Social, environmental and cultural processes}

The evolution of social processes, as well as environmental and cultural themes, inevitably brings potential change to mental health and to the understanding and management of mental illnesses. War, dislocations, psychological damage through conflict, loss of social status, breakdown of social environments and support networks, along with stresses such as the global financial crisis, may all make people more vulnerable and in need of psychiatric care. Strong social cohesion and social capital may protect and enhance health and health care.

Negative change may result from social exclusion and all its associated wounds, as shown by the experience of Indigenous Australians, and also by indigenous people in many other countries. Progressive socially and politically driven changes, including recognition of culture, have provided positive frameworks, but the ways forward (Swan and Raphael 1995) constitute a long journey. In Australia's case, special initiatives such as 'Closing the Gap' are making improvements, and recognition, respect, the Apology, education, and valuing this oldest surviving culture will all help to build positive social and emotional wellbeing, and promote culturally appropriate treatments for those with mental illnesses.

New displaced populations such as refugees (the 'boat people') also highlight needs for change for better mental health. Adverse social determinants, societal discrimination, and the politics of power can lead to negative change processes, or challenge change to contribute to positive mental health outcomes.

Stigma and discrimination have been a focus for strategies for change, with many agencies promoting programs to counter them. The UK's well funded, multifaceted program for mental health, 'Time to Change', has recently been evaluated, highlighting the importance of these issues as obstacles for mental health programs. Though change for the better was achieved, there are still many ongoing issues. Like all change intentions, there are multifaceted outcomes (Sartorius 2013).

Social and cultural processes can also define what illness is and what it is acceptable to do about it. This is particularly the case for psychiatry problems because of the overlap and interfaces with life, highlighted earlier in this chapter. The influence of social and cultural processes was discussed by psychiatric and anthropological colleagues (Hansen et al. 2013) exploring from social determinants, social science and population health points of view whether there were changes in environmental factors causing an actual increase in mental health problems; whether diagnostic criteria were applied differently; or whether diagnostic criteria could have been written in a way that could include people who did not have pathology - just the human spectrum. 
Hansen and colleagues from the New York University Langone Health Center intend to set up a new independent research and review process to focus on such perspectives on mental health.

\section{Technological advances, knowledge expansion and communication}

The ability to spread knowledge through the internet, Google, the e-revolution, and the breadth of media coverage has been of value across many domains relevant to health and mental health. There is now constant exposure, in Australia at least, to information about mental illnesses and the range of associated problems. Technological advances have also provided platforms for assessing and treating these disorders (Looi and Raphael 2007).

Furthermore, technological changes in and of themselves have stimulated, threatened and universally engaged the human population across all age groups. The change processes are massive in terms of access, connectivity and instantaneity of information. These are all changing mental health in new ways, for instance with ' $i$ ' disorders (Rosen 2012), Facebook support and information to deal with emergency threats (Taylor et al. 2012), trauma help lines, and treatments facilitated by technologies of the internet. Some exposures are said to create problems, for instance children's repeated exposure to very traumatic experiences on television replays, or addiction to violent computer games.

Technological advances mean that social and physical opportunities and environments will continue to change rapidly, perhaps in unanticipated ways, and technologies can now influence the nature and functioning of brain and mind. The two-way neurology-engineering models, and real world braintechnology interfaces are examples for the future, as are brain-computer interfaces.

These changes continue to escalate, alongside many other questions of demand and response. Instantaneity will challenge human adaptation, and mental health and wellbeing - for better and for worse, but inevitably for the future.

\section{The voices of the people}

'The people' are many. They are those most severely affected and their families the consumers and the carers. Their voices grow progressively stronger and they are being heard. In addition, studies examining the 'burden of disease' show the importance of psychiatric problems, with depression alone being a major component. This has been a driver that has influenced governments, funding bodies and organisations nationally. Ongoing studies in Australia, both those 
examining the prevalence and impairments associated with psychiatric illnesses, and reported studies of the broader social and economic 'burden', bring a strong rationale for the need to better address these problems.

Voices that are the most potent, the voices that have driven change, are those of people themselves. The power and expertise of the consumer movement in mental health in Australia has been supported by the Department of Health's Mental health statement of rights and responsibilities (1991), which has developed over time. Voices of advocates and supporting organisations arguing for representation, respect and formally having a say, as well as presenting stories of cost, burden, and human experience, have all been potent for change. They have rightly demanded recognition of the need for effective safe services, access, quality care, and support systems for carers. Non-government agencies, for instance SANE Australia (a leading national mental health non-government organisation) and the Consumer Advisory Group of the Australian state of New South Wales and such bodies in other states and territories have added their contributions. These voices now have formal and required representation at all levels of service, government, and community, with mottos such as 'Nothing about us without us'. Other consumer movements monitoring the nature and bases of care systems are also strong, influential and easily identifiable.

All these voices, those of the people, professionals, the public, the scientists and the wider community, have driven and are ongoing drivers of change.

\section{Conclusions}

Many changes have happened to improve the treatment and care of people with mental illnesses across the human lifespan and, as described above, there have been many drivers of such changes - most powerfully the voices of the many communities involved. Change is continuous, evolving, and developing with a growing world of people who will face differing future challenges: climate change, technological advances and inventions that will transform our lives, and perhaps even life on Mars (Kaku 2011).

There is more change to come. The true challenges for mental health are to resist the seductions of conservatism that hold us to familiar ideologies, and to tune to opportunities for change - change that involves risk, hopes to prevent illness, to build with courage and strength, to listen and learn from the multiple expertises within and outside this specialised field, for the future.

Many changes have occurred for mental health, mental illnesses and psychiatrybut many more are needed. These include the capacity to know and manage issues of change such as: 
- Are mental health problems getting worse - more prevalent, more severe? Have they changed; if so, how and why?

- Why is it so difficult to change to prevention, early intervention and treatment with cure for mental illnesses?

- Why is mental health still so separated from health and identified by the challenges of 'serious mental illness'?

- Why are there no answers to questions about why so many children and young people have mental illness, and what changes will make things better?

- And why are we not able to ride the waves of change affecting the human condition, the spectrum of health and mental health problems, for more positive futures for our children?

\section{References}

Australian Department of Health (1991) Mental health: statement of rights and responsibilities (see www.health.gov.au/internet/main/publishing.nsf/Conte nt/1A5FE569B1D0E391CA257BF0001A9342/\$File/rights.pdf).

Engel, GL (1961) Is grief a disease?: a challenge for medical research. Psychosomatic Medicine 23 (January): 18-22.

Freud, S (1917) Mourning and melancholia.The Standard Edition of the Complete Psychological Works of Sigmund Freud, vol. XIV (1914-1916): On the history of the psycho-analytic movement, Papers on metapsychology and other works, 237-258s.

Hansen, HB, Donaldson, Z, Link, BG, Bearman, PS, Hopper, K, Bates, LM, Cheslak-Postava, K, Harper, K, Holmes, SM, Lovasi, G, Springer, KW and Teitler, JO (2013) Independent review of social and population variation in mental health could improve diagnosis in DSM revisions. Health Affairs 32 (May): 984-93.

Hortwitz, AV and Wakefield, JC (2007) The loss of sadness. Oxford University Press, New York.

Kaku, M (2011) Physics of the future: the interventions that will transform our lives. Penguin Books, London.

Licinio, J (2013) Translational psychiatry: leading the transition from the cesspool of devastation to a place where the grass is really greener. Translational Psychiatry 1: el; doi:10.1038/tp.2011.3 2158-3188. 
12. Mental Illness and Psychiatry Have Seen Substantial Change-But There is Still a Long Way To Go

Lindemann, E (1944) The symptomatology and management of acute grief. American Journal of Psychiatry 101: 141-8.

Looi, J and Raphael, B (2007) Reflection on therapy in the era of the internet. Australasian Psychiatry 15(4) August: 334-7.

McGorry, P (2010) Early psychosis guidelines writing group, Australian clinical guidelines for early psychosis, 2nd ed. Orygen Youth Health, Melbourne.

McGorry, P (2011) Early psychosis feasibility study report. Prepared by Orygen Youth Health Research Centre.

Rosen, L (2012) iDisorder: the psychology of technology. Palgrave McMillan.

Sanders, M (2013) Triple P-Positive Parenting Program: review of evidence. Parenting and Family Support Centre. University of Queensland, Brisbane, March.

Sartorius, N (1994). 'The Rolling Front End'. Talk presented at Psychiatry Meeting, Indianapolis.

Sartorius, N (2013) Time to change. British Journal of Psychiatry 202: S108-09.

Swan, P and Raphael, B (1995) 'Ways Forward': national consultancy report on Aboriginal and Torres Strait Islander Mental Health, Parts $l$ and $l l$. Commonwealth of Australia, Canberra.

Taylor, M, Wells, G, Howell, G and Raphael B. (2012) The role of social media as psychological first aid as a support to community resilience building: a Facebook case study from 'Cyclone Yasi Update'. Australian Journal of Emergency Management 27(1): 20-6. 
This text is taken from Change! Combining Analytic Approaches with Street Wisdom, edited by Gabriele Bammer, published 2015 by ANU Press, The Australian National University, Canberra, Australia. 